\title{
Degree of agreement between AOAC-based dietary fibre intake and Englyst-based dietary fibre intake in the UK Women's Cohort Study (UKWCS)
}

\author{
M. Aldwairji, C. Orfila and V. J. Burley \\ School of Food Science and Nutrition, University of Leeds, Leeds, LS2 9JT, UK
}

\begin{abstract}
Nutrition guidelines encourage dietary fibre intake with the aim to prevent nutrition-related chronic diseases ${ }^{(1)}$. Currently, official methods of analyses named Association of Official Analytical Chemists (AOAC) 985.29 and $991.43^{(2)}$ are the methods adopted for total dietary fibre (TDF) analysis, for food composition tables and food labelling in most European countries and in the U.S.A ${ }^{(1)}$. However, in the UK, the Englyst method ${ }^{(3)}$ is used to determine non-starch polysaccharides (NSP) for food composition tables. The variability across different countries in recommended amounts of dietary fibre consumption can be partly explained by the methods used to measure dietary fibre. Higher dietary fibre values measured by the AOAC method compared to the Englyst method are explained by the wider range of components measured by the AOAC method, such as resistant starch and lignin ${ }^{(1)}$. However, variation in dietary fibre analysis methods presents a challenge for epidemiologists who investigate the association between dietary fibre intake and the risk of chronic disease. The aim of this analysis was to investigate the relationship between the two analytical methods in estimating dietary fibre in the UK Women's Cohort Study (UKWCS) ${ }^{(4)}$ with a view to facilitation of future epidemiological analyses of diet: disease associations.

Between 1995 and 1998, 35,372 women aged 35-69 years completed a 217-item self-administered food frequency questionnaire (FFQ), and these women formed the UKWCS. AOAC-total dietary fibre values were added to the UKWCS database for all food items listed in the FFQ. Values were obtained from a variety of sources, including the UK food composition tables ${ }^{(5)}$, a British Nutrition Foundation review $^{(1)}$ and the EuroFIR database (data accessed from European Food Information Resource database; available at http://esearch.eurofir.org/ to registered users). Non-starch polysaccharide values had been assigned to FFQ items previously. Thus, daily dietary fibre intakes expressed as both NSP and AOAC were derived for all cohort members by multiplying the frequency of consumption of each FFQ item by the item portion size and the estimated fibre content of each FFQ item. A comparison of fibre intakes expressed as NSP and AOAC values was undertaken. Cohort participants were split into quintiles of intake of both types of dietary fibre and extent of misclassification of individuals into different quintiles was assessed using a weighed Kappa. Linear regression was used to predict participant level AOAC-derived dietary fibre intakes from Englyst dietary fibre values.

As anticipated, mean (SD) AOAC-derived dietary fibre intake was significantly higher than the Englyst-derived dietary fibre intake (39.0 (15.5) vs. 26.0 (10.5) g/day, respectively; $p<0.001)$. The weighed kappa $(\kappa)$ analysis found an agreement of $96.2 \%$, with a $\kappa=0.9$, which is considered perfect agreement in terms of allocation to quintiles using the two fibre analysis methods. This indicates that the majority of the cohort participants were placed in the same fibre quintile, regardless of the method of fibre analysis used. None of the consumers were misclassified in the opposite extreme quintiles. The linear regression indicated that each $1 \mathrm{~g}$ increase in NSP was associated with a $1.5 \mathrm{~g}$ increase in AOAC dietary fibre. This is somewhat higher than a previously published ratio of $1.3^{(1)}$. These analyses suggest that for the conduct of a meta-analysis that compares risk of disease in the highest versus the lowest quintiles of dietary fibre intake the method of fibre analysis employed is of minor importance. High consumers will be categorised as such regardless of the method used. However, for a dose-response meta-analysis, pooling of results using different fibre analysis methods would be inappropriate.
\end{abstract}

1. Lunn J \& Buttriss L (2007) Nutr Bulletin 32, 21-64.

2. AOAC (1995) Official Methods of Analysis, 16th ed. AOAC International, Gaithersburg, MD, 1995.

3. Englyst H, Wiggins HS \& Cummings JH (1982) Analyst 107, 307-318.

4. Cade JE, Burley VJ, Greenwood DC et al. (2004) Public Health Nutr 7, 871-878.

5. Food Standard Agency (2002) McCance and Widdowson's The Composition of Foods. Cambridge: Royal Society of Chemistry. 